\title{
Retrieval of structure functions of air temperature and refractive index from large eddy simulations of the atmospheric boundary layer
}

\author{
Chris Wilson $^{\mathrm{a}}$, Alexander M. J. van Eijk ${ }^{\mathrm{b}, \mathrm{c}}$, Evgeni Fedorovich ${ }^{* a}$,

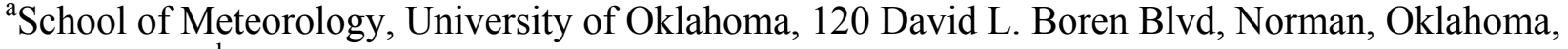 \\ USA 73072; ${ }^{\mathrm{b}} \mathrm{TNO}$, P.O. Box 96864, $2509 \mathrm{JG}$ The Hague, the Netherlands; ${ }^{\mathrm{L}} \mathrm{LUNAM}$ Université, \\ Ecole Centrale de Nantes, LHEEA UMR CNRS 6598, 1 rue de la Noë, 44300 Nantes, France
}

\begin{abstract}
A methodology is presented to infer the refractive-index structure function parameter and the structure parameters for temperature and humidity from numerical simulations of the turbulent atmospheric convective boundary layer (CBL). The method employs spatial and temporal averaging of multiple realizations of the CBL flow field reproduced by a large-eddy simulation (LES) of the atmosphere. The $C_{n}^{2}$ values yielded by LES-based approach agree fairly well with $C_{n}^{2}$ values predicted by the Monin-Obukhov similarity theory. In this respect, the $C_{n}^{2}$ retrieval from the LES data is promising for evaluating the vertical profile of $C_{n}^{2}$ throughout the entire CBL. Under the considered CBL conditions and for the selected optical wavelength of $0.55 \mu \mathrm{m}$ the value of $C_{n}^{2}$ was found to be dominated by the $C_{T}^{2}$ contribution in the first few hundred meters above the surface, whereas the $C_{T q}$ contribution became significant aloft.
\end{abstract}

Keywords: turbulence, boundary layer, numerical simulation, wave propagation, structure function, structure parameter.

\section{INTRODUCTION}

Advanced optical imaging sensors and optical communication systems combine high resolution and high sensitivity. While this potentially allows for long-range applications, the performance of these systems is often hampered by the intervening of the Earth's atmosphere. Apart from transmission losses due to absorption and scattering of radiation by atmospheric constituents, the signal is degraded by atmospheric turbulence generated by wind (shear) and/or by convective processes due to temperature and/or humidity inhomogeneities. Turbulence effects cause intensity fluctuations and directional variations of the propagating radiation, which result in scintillation, blurring, and image dancing at the receiver. These effects decrease the spatial resolution of the image ${ }^{1}$ or the quality of the communication $\operatorname{link}^{2}$. On the other hand, scintillation effects may be used to our advantage, e.g., in the detection of point targets, as the peak levels signals may exceed the mean signal level by a factor 3 or more ${ }^{3}$.

Optical turbulence is caused by small-scale and rapid variations of the atmospheric refractive index which, in turn, are induced by variations of pressure, temperature and humidity along the path of observation. The effects of atmospheric turbulence are generally considered ${ }^{5}$ in terms of the second-order refractive index structure function given by

$$
\overline{(\partial n)^{2}}(\mathbf{r}, t)=\overline{[n(\mathbf{x}, t)-n(\mathbf{x}+\mathbf{r}, t)]^{2}},
$$

where the overbar on represents ensemble averaging (which in practical terms is typically substituted by the spatial averaging), $n$ is the refractive index, $\mathbf{r}$ the separation vector, $\mathbf{x}$ the position vector, and $t$ is time. Within the framework of Kolmogorov's hypotheses ${ }^{6}$ for the inertial-subrange turbulence, the structure function takes the form

$$
\overline{(\partial n)^{2}}=C_{n}^{2} r^{2 / 3}
$$

where $r$ is the separation vector magnitude (separation distance) and $C_{n}^{2}$ is the refractive index structure-function parameter (also called the structure parameter). Analogously to eq.(2), the structure parameters for temperature and humidity $C_{T}^{2}$ and $C_{q}^{2}$ can be defined in terms of structure functions of the turbulent fluctuations of potential temperature $\theta$ and humidity $q, \overline{(\partial \theta)^{2}}$ and $\overline{(\partial q)^{2}}$, respectively.

\footnotetext{
*fedorovich@ou.edu; phone 1405 325-1197; fax 1405 325-7689; som.ou.edu
} 
By neglecting pressure effects on the fluctuations of the refractive index ${ }^{4}$ and by assuming that the fluctuations of the refractive index can be described by a linear combination of the fluctuations in temperature and humidity ${ }^{4,7}$, i.e., as

$$
\partial n=A \partial \theta+B \partial q,
$$

eq.(2) can be rewritten as ${ }^{8}$

$$
C_{n}^{2}=\left(\frac{\partial n}{\partial T}\right)^{2} C_{T}^{2}+\left(\frac{\partial n}{\partial q}\right)^{2} C_{q}^{2}+2\left(\frac{\partial n}{\partial T} \frac{\partial n}{\partial q}\right) C_{T q}=A^{2} C_{T}^{2}+B^{2} C_{q}^{2}+2 A B C_{T q},
$$

where $C_{T q}$ is the joint temperature-humidity structure-function parameter, defined through $\overline{(\partial \theta \partial q)} r^{-2 / 3}$.

The refractive index structure-function parameter $C_{n}^{2}$ can be used to quantify turbulence effects on the radiation propagation such as scintillation, blur, and beam wander. As an example, the standard deviation $\sigma_{I}$ of the fluctuations in signal intensity $I$, within the Rytov approximation, is given by ${ }^{9}$

$$
\sigma_{I}^{2}=\frac{\left\langle[I-\langle I\rangle]^{2}\right\rangle}{\langle I\rangle^{2}}=\exp \left(4 \sigma_{\chi}^{2}\right)-1 \approx 4 \sigma_{\chi}^{2},
$$

where the angle brackets denote temporal averaging and the variance $\sigma_{\chi}^{2}$ of the electromagnetic propagation constant, for plane waves and a point receiver, is given by ${ }^{9}$

$$
\sigma_{\chi}^{2}(R)=0.56 k^{7 / 6} \int_{0}^{R} C_{n}^{2}(\xi)(R-\xi)^{5 / 6} d \xi,
$$

where $k$ is the wavenumber ( $k=2 \pi / \lambda$, with $\lambda$ the wavelength), $R$ is the path length and $\xi$ is the distance along the path. Equation (6) shows that turbulence near the receiver does not contribute significantly to the scintillation, which emphasizes the need to evaluate the spatial variance of $C_{n}^{2}$ along the propagation path and not to be restricted to a value obtained at the location of the receiver.

Over the years, a multitude of models have attempted to describe the vertical variation of the structure-function parameters. One of the earliest approaches was based on similarity theory. It modeled the daytime falloff of $C_{n}^{2}$ with height as a $-4 / 3$ power law, using the near-surface value of $C_{n}^{2}$ as a gauge ${ }^{10,11}$. On the basis of experimental data, Hufnage $^{12}$ and Valley ${ }^{13}$ introduced an altitude falloff dependent on the average wind speed between 5 and $20 \mathrm{~km}$ altitude, although most users of the Hufnagel-Valley model apply it with standard values for wind speed and $C_{n}^{2}$ at the surface. An explicit dependence of the structure-function parameters on meteorological variables in the near-surface portion of the atmosphere, the so-called atmospheric surface layer, was established using the Monin-Obukhov similarity theory ${ }^{14}$, which resulted in ${ }^{4,15-17}$

$$
C_{\varphi}^{2}=\varphi_{*} z^{-2 / 3} f_{\varphi}\left(\frac{z}{L}\right), \quad C_{T q}=q_{*} \theta_{*} z^{-2 / 3} f_{T q}\left(\frac{z}{L}\right),
$$

where $\varphi$ can be $T, q$, or $n, \varphi_{*}$ denotes the corresponding surface-layer turbulence scale, $f_{\varphi}$ the corresponding stability function, and $L$ the Obukhov length (scale). The turbulence scales and Obukhov length are calculated from bulk meteorological parameters, such as the wind speed, temperature, and humidity, which can be relatively easy obtained insitu.

While the explicit form of the stability functions $f_{\varphi}$ has been the subject of extensive debate, the Monin-Obukhov similarity theory (MOST) formalism outlined above provides reasonable estimates of the vertical profiles of the structure-function parameters in the atmospheric surface layer. Because the calculation is based on data from in-situ meteorological measurements, it is less straightforward to obtain the full 3-D inhomogeneous field $C_{n}^{2}(x, y, z)$ and horizontal homogeneity of the surface layer (in statistical sense) is generally assumed. This shortcoming can be partly overcome by employing bi-static experimental techniques (e.g., scintillometry) that characterize turbulence along a path. However, these instruments yield path-averaged quantities ${ }^{18}$ that are not easily decomposed in the full 3-D field of $C_{n}^{2}(x, y, z)$. A solution may be offered by the advent of numerical weather prediction (NWP) models. Traditionally applied on numerical grids with a horizontal spacing of the order of tens of kilometers, recent advances in regional NWP allow for spacing down to the order of a few kilometers. Particularly, the community Weather Research and Forecasting (WRF) model ${ }^{19}$ was shown to reasonably accurately represent the atmospheric near-surface flow under convective conditions $^{20}$, which opens an avenue to drive the MOST calculations, eq. (7), by gridded WRF model data and thereby obtain $C_{n}^{2}(x, y, z)$ with the corresponding horizontal resolution. This approach is not limited to evaluating $C_{n}^{2}(x, y, z)$ on 
the basis of the actual meteorological conditions, but can also be applied to predict sensor image quality or the strength of optical links under forecasted meteorological conditions.

A more detailed description of turbulence can be achieved with the high-resolution computational fluid dynamics (CFD) technique generally known as large eddy simulation (LES ${ }^{21,22}$. LES considers the turbulent flow as a superposition of larger and smaller flow motions. The LES numerical approach resolves the larger, energy containing motions explicitly, while the effects of smaller, the so-called subgrid motions, are modeled. LES codes resolve fields of fundamental meteorological parameters (wind velocity, temperature, humidity) in the atmospheric boundary-layer flows down to spatial scales of the order of meters. These parameters can be entered in the calculation of the refractive index, which thus allows evaluating its spatial variability, in terms of $C_{n}^{2}$, on a scale of meters.

This paper reports application of the LES technique to direct evaluation of structure functions of potential temperature, specific humidity, and refractive index in the turbulent atmospheric convective boundary layer (CBL). The LES estimates of structure-function parameters for the lower portion of the CBL will be compared to the structure parameters obtained with the traditional approach ${ }^{4}$ based on MOST. Most of results from this comparative study have already been reported in literature ${ }^{23}$ but are presented here to bridge the gap between the boundary-layer meteorology and the optical communication and propagation communities.

\section{EXPERIMENTAL DESIGN}

The simulations reported here were performed using the LES code of the School of Meteorology (SoM) of the University of Oklahoma (OU), henceforward denoted as OU-LES. The OU-LES code has been shown to confidently reproduce CBL flow structure in terms of mean-flow parameters and turbulence statistics up to the third order ${ }^{24,25}$. The considered OU-LES setup employed a numerical grid with spatially uniform spacing of $10 \mathrm{~m}$ in a numerical domain with dimensions $(X, Y, Z)=(2.56 \mathrm{~km}, 2.56 \mathrm{~km}, 3.00 \mathrm{~km})$. The horizontal dimensions of domain were proven, through numerical experiments, adequate for the reproduction of turbulence statistics in a moderately sheared CBL within the desired target scale ranges (up to several hundreds of meters).

The simulations focused on the CBL case that was observed at the Lamont (Oklahoma) site of the Atmospheric Radiation Measurement (ARM) Program on 31 May 2009. This ARM site is heavily equipped with diverse meteorological instrumentation, including an eddy correlation flux measurement system ${ }^{26}$ that provided the surface sensible and latent heat fluxes (30-minute averages) to drive the OU-LES. Geostrophic wind profiles (representing in the LES the large-scale pressure-gradient forcing) were retrieved from the Rapid Update Cycle (RUC) model ${ }^{27}$ on an hourly basis. To account for other features of atmospheric variability on scales larger than the scale of the LES domain, the LES solutions at each time step were nudged ${ }^{20}$ towards the profiles of horizontal velocity components, potential temperature, and specific humidity available from the RUC model. The nudging time constant was set to $3600 \mathrm{~s}$, which is larger than the typical CBL overturn time scale, but smaller than the time scale of boundary layer diurnal variation. Thus, the nudging is not expected to noticeably affect turbulent fluctuations within the scale ranges of interest (time scales up to few minutes and length scales up to several hundreds of meters).

The refractive index $n$ for visible wavelengths is calculated from ${ }^{4,28}$

$$
n=1+10^{-6}\left\{m_{1}(\lambda) \frac{p}{T}+\left[m_{2}(\lambda)-m_{1}(\lambda)\right] \frac{q p}{0.62197 T(1+0.61 q)}\right\},
$$

where $\lambda[\mu \mathrm{m}]$ is the wavelength, $p[\mathrm{hPa}]$ is atmospheric pressure, $T[\mathrm{~K}]$ is absolute temperature, and $q[\mathrm{~kg} / \mathrm{kg}]$ is specific humidity. Parameters $m_{1}$ and $m_{2}$ are given by

$$
\begin{gathered}
m_{1}(\lambda)=23.7134+\frac{6839.397}{130-\lambda^{-2}}+\frac{45.473}{38.9-\lambda^{-2}}, \\
m_{2}(\lambda)=64.8731+0.58058 \lambda^{-2}-0.0071150 \lambda^{-4}+0.0008851 \lambda^{-6} .
\end{gathered}
$$

Squared differences of $n$ for the evaluation of $C_{n}^{2}$ by eq. (2) were calculated in space as functions of separation distances in $x, y$, and $z$. The separations were integer multiples of the spatially uniform LES grid spacing $\Delta(=10 \mathrm{~m})$.

For the $x$-horizontal direction, differences $n(t, z, y, x)-n\left(t, z, y, x+x_{i}\right)$ with $x_{i}=\Delta$ were calculated at all individual points $(x, y)$ in a horizontal plane for a given $z$ and $t$. If $x+x_{i}$ corresponded to a location outside the LES-domain, the 
calculation was skipped. Individual contributions were added to a running sum, from which the average was computed after completion of the process. The process was then repeated with a new separation $x_{i}=2 \Delta$, and so on, until the separation distance became larger than $1000 \mathrm{~m}$. The entire procedure was repeated for the next height $z$, and after completion of the height loop, for the next time $t$. Finally, the horizontal plane averages were additionally averaged in time, which yielded a direct estimate of the refractive-index structure function in the $x$ direction:

$$
\left.\overline{(\delta n)^{2}}\right|_{x}(z, i \Delta)=\left\langle\left.(\delta n)^{2}\right|_{x}\left(t, z, x_{i}, y, x\right)\right\rangle_{x, y, t},
$$

where $i \Delta=x_{i}$ and the brackets signify the averaging over horizontal planes and time. The time averaging was performed over a one hour timeframe (for this study, 19:00 through 20:00 UTC on 31 May 2009), corresponding to approximately 3600 flow realizations of the simulated CBL flow field.

The same procedure was followed in the calculation of the structure function in the $y$-horizontal direction, but a slight difference was made for evaluation of $\overline{(\delta n)^{2}}$ in the vertical direction. In this case, the influence of the mean vertical gradient of $n$ in structure function calculations was removed by taking

$$
\left.(\delta n)^{2}\right|_{z p}\left(t, z, z_{k}, y, x\right)=0.5\left(\left[n^{\prime}(t, z, y, x)-n^{\prime}\left(t, z+z_{k}, y, x\right)\right]^{2}+\left[n^{\prime}(t, z, y, x)-n^{\prime}\left(t, z-z_{k}, y, x\right)\right]^{2}\right),
$$

where $z_{k}$ is the separation distance in the $z$ direction and $n^{\prime}$ denotes the fluctuations of $n$ from its horizontal plane averages. In this way, $\left.\overline{(\delta n)^{2}}\right|_{z p}$ was calculated as the average of squared differences of $n^{\prime}$ in positive and negative directions.

Structure functions $\overline{(\delta n)^{2}}$ evaluated in the above described manner were first examined for the presence of inertial subranges of separation distances in order to justify application of eq.(2) for the calculation of $C_{n}^{2}$ and then, if the conditions were met, $C_{n}^{2}$ values were computed. The same methodology was employed to post-process LES potential temperature and humidity fields, which yielded $C_{T}^{2}, C_{q}^{2}$, and $C_{T q}$ by using equations analogous to eq.(2). By plugging these three structure-function parameters in eq.(4), $C_{n}^{2}$ could also be evaluated indirectly, based on the assumed linear relationship (3). For visible wavelengths, the explicit expressions for $A$ and $B$ are ${ }^{4}$

$$
A=-10^{-6} m_{1}(\lambda) \frac{p}{T^{2}}, \quad B=4.6150 \cdot 10^{-6}\left[m_{2}(\lambda)-m_{1}(\lambda)\right] .
$$

\section{RESULTS}

The OU-LES was run in two configurations. The first one, denoted as LES10, was a simulation of the actually observed CBL at the ARM site on 31 May 2009. LES10 represented the main features of the observed CBL rather realistically,

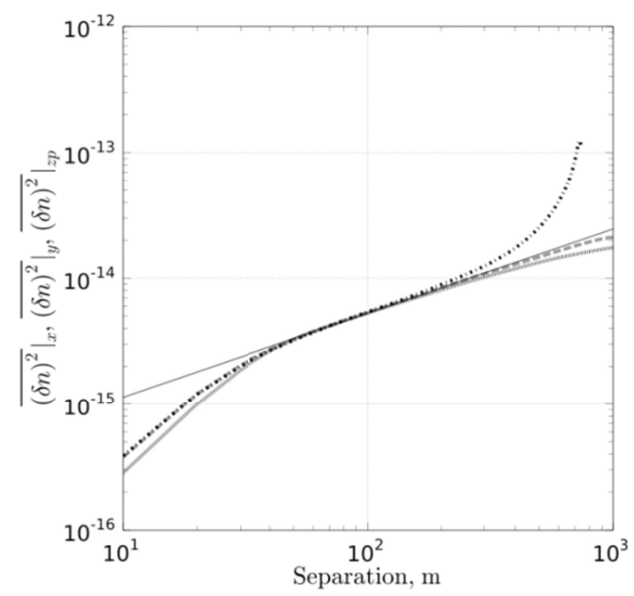

Figure 1: Directly evaluated second-order refractive index structure functions $\overline{(\partial n)^{2}}$ in $x$ - (dashed line), $y$ - (dotted line) and $z$ (dashed-dotted line) direction. The straight black line denotes the $r^{2 / 3}$ dependence. 
e.g., it exhibited the well-mixed CBL-interior below a capping inversion with jumps in the plane-averaged $q$ and $T$ at $1500 \mathrm{~m}^{23}$, with turbulence statistics throughout the layer also looking quite reasonable. Since the surface latent heat flux was rather weak, the refractive index variability in LES10 was predominantly caused by the temperature fluctuations. In the second configuration, denoted by LES10EMF, OU-LES was run with the surface latent heat artificially increased by factor of five compared to the observed one. In all other respects this case was a replica of the LES10 configuration. The idea of running LES10EMF was to evaluate contributions of the humidity-related structure parameters $C_{q}^{2}$ and $C_{T q}$ to $C_{n}^{2}$.

Equations (1) and (8) indicate that the refractive-index structure functions $\overline{(\partial n)^{2}}$ depend on height and separation distance. The dependence on separation distance is demonstrated in Figure 1 which shows the refractive-index structure functions $\overline{(\partial n)^{2}}$ directly evaluated from the LES for the $x$-, $y$-, and $z$-direction using eq.(11) and its analogs. These functions were evaluated from the LES10EMF run at a height of $745 \mathrm{~m}$ (that is, within the mixed region of the CBL), and for a refractive index corresponding to a wavelength of $0.55 \mu \mathrm{m}$. The counterpart structure functions $\overline{(\partial n)^{2}}$ evaluated indirectly from the LES potential temperature and humidity fluctuations, i.e., through $\overline{(\partial \theta)^{2}}$ and $\overline{(\partial q)^{2}}$ according to eq.(4), are not shown, but exhibit similar behavior.

Figure 1 shows the range of separation distances (approximately from 40 to $200 \mathrm{~m}$ ) for which the structure functions exhibit the $r^{2 / 3}$ dependence expected for the inertial subrange ${ }^{6}$. Only for these separations does the concept of structure function parameters, implied by eq.(2), make sense. At separation distances larger than $200 \mathrm{~m}$, the structure function in the $z$-direction notably deviates from the $r^{2 / 3}$ dependence. This deviation indicates that squared differences of the refractive index at the corresponding separation distances are calculated between the reference point at $745 \mathrm{~m}$ and a point that is located in portions of the CBL (surface layer, entrainment zone) with essentially different turbulence properties or in the practically turbulence-free atmosphere above the CBL (it is reminded here that the vertical domain of the LES extends up to $3000 \mathrm{~m}$ ). The upper limit of $200 \mathrm{~m}$ for the vertical separation distance is thus expected to be a function of the height at which $\overline{(\partial n)^{2}}$ is evaluated. Figure 1 also shows the falloff of the structure functions at separation distances below $40 \mathrm{~m}$. Such behavior can be attributed to the finite resolution of the LES: only the fluctuations that are larger than the grid spacing size are numerically resolved to the full extent, while fluctuations that are comparable in size with the grid spacing are attenuated due to numerical and subgrid-model effects. This reduces the spatial variability in the refractive-index field at smaller separations. Additional simulations (not shown) demonstrated that the lower limit of the separation distance is typically about $4 \Delta$, where $\Delta$ is the LES grid spacing.

Figure 2 shows the vertical profiles of $\left.C_{n}^{2}\right|_{x}$, evaluated by the direct (eqs. 8-11, solid line) and indirect (eq. 4, dashed line) methods. The separation distance was $60 \mathrm{~m}$, and as before, the structure parameters were evalluated from the LES10EMF run for the wavelength of $0.55 \mu \mathrm{m}$. Profiles of $C_{n}^{2}$ in the $y$ - and $z$-direction are quite similar to the $C_{n}^{2}$ profile in $x$ -

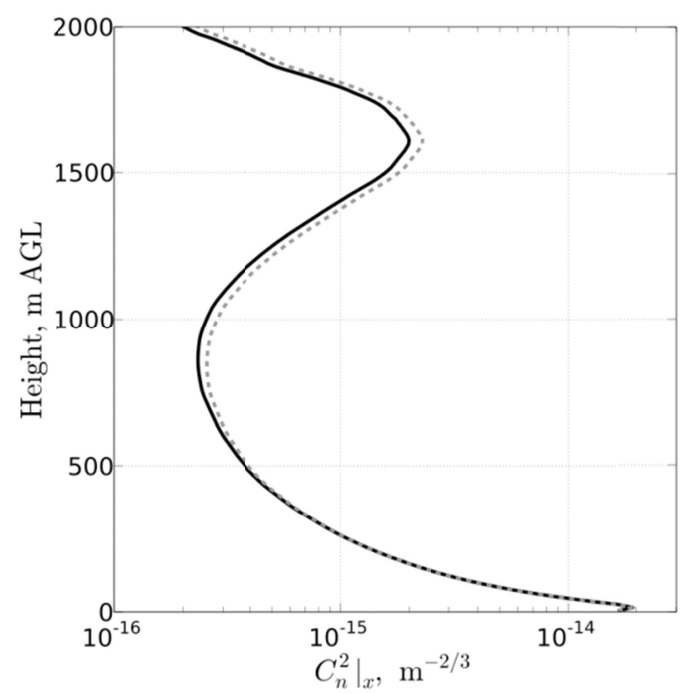

Figure 2: Vertical profiles of $C_{n}^{2}$ in the $x$-direction, evaluated by the direct (solid line) and indirect (dashed line) methods. 


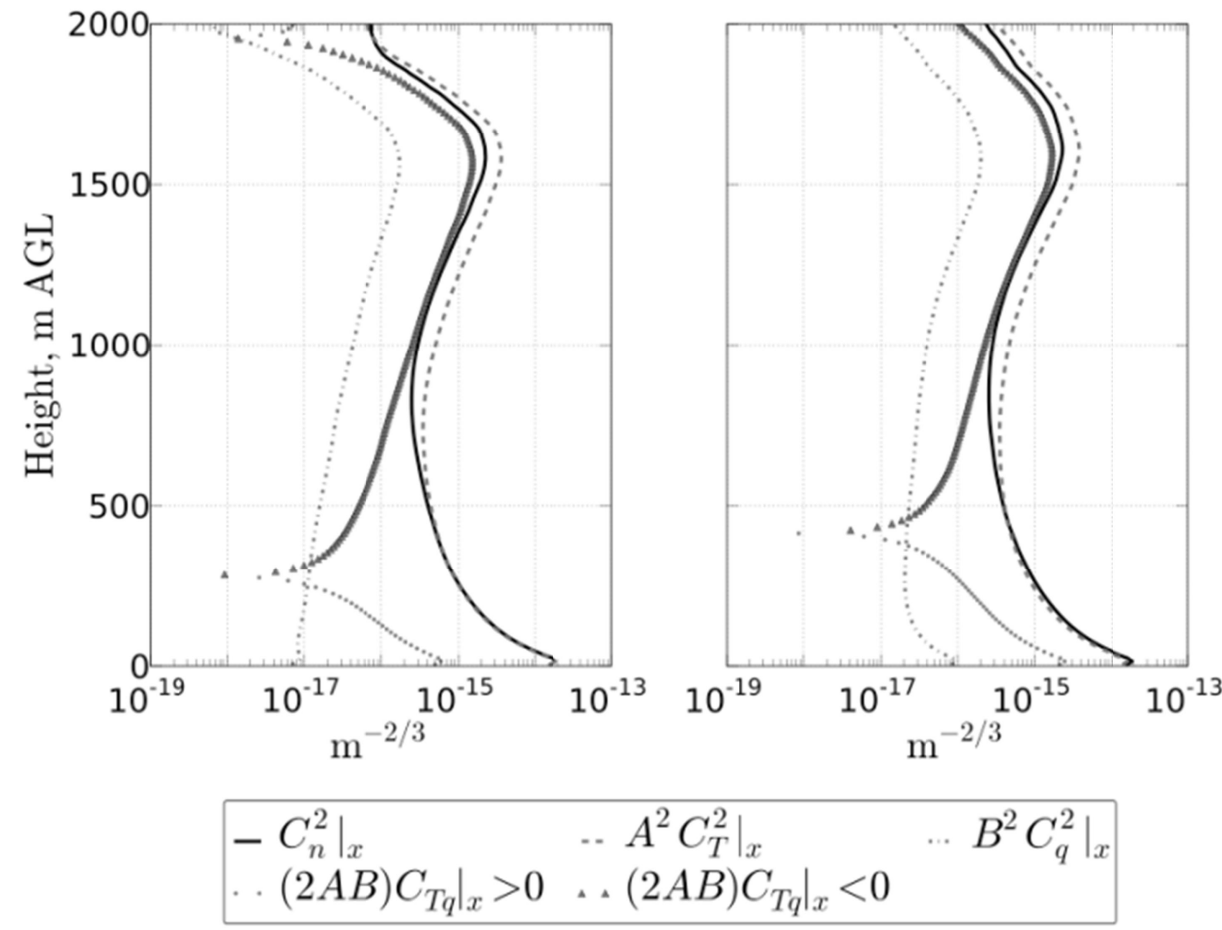

Figure 3: Vertical profile of $\left.C_{n}^{2}\right|_{x}$, and individual contributing terms in eq.(4) for simulations LES10 (left) and LES10EMF (right).

direction and are not shown. One may see that $C_{n}^{2}$ values calculated by the direct and indirect methods start to deviate at higher altitudes, above $500 \mathrm{~m}$, where the indirect method overestimates the value of $\left.C_{n}^{2}\right|_{x}$ by several percent. This could be a result of neglecting the pressure term and/or cross-terms between potential temperature and specific humidity in eq.(3), which approximates $\partial n$ for the indirect method.

Figure 3 shows the vertical profiles of $\left.C_{n}^{2}\right|_{x}$, for a separation distance of $60 \mathrm{~m}$ and a wavelength of $0.55 \mu \mathrm{m}$, retrieved from the two simulations with different surface latent heat flux. In both cases $\left.C_{n}^{2}\right|_{x}$ was obtained by the indirect method (eq. 4). The figure also shows the individual contributing terms $\left.C_{T}^{2}\right|_{x},\left.C_{q}^{2}\right|_{x}$, and $\left.C_{T q}\right|_{x}$. Once again, results for the $y$ - and $z$-directions were very similar and are not shown. For both LES configurations, the $C_{T}^{2}$ term dominates the $C_{n}^{2}$ budget in the lower part of the CBL. Even the increased surface latent heat flux in LES10EMF does not increase $C_{q}^{2}$ sufficiently to become the dominant contributor. Figure 3 also illustrates the height at which the $C_{T q}$ term changes sign: for LES10EMF this change takes place at higher altitude than for LES10. The variation of the transition height with the strength of the surface latent heat flux is important, because at higher altitudes in the $\mathrm{CBL} C_{T q}$ represents a significant contribution to $C_{n}^{2}$. Hence, the dominance of the $C_{T}^{2}$ term is reduced here.

Figure 4 (next page) presents a comparison of the $C_{n}^{2}$ profile obtained by MOST, following Andreas ${ }^{4}$, with the $C_{n}^{2}$ profiles obtained by the direct method from simulation LES10EMF. The indirect method yielded similar results that are not shown here. Since MOST is only applicable in the surface layer, the comparison does not extend above 125 meters. For this domain, MOST predicts a higher value than the LES, which may be partially due to the inability of the LES to fully resolve temperature fluctuations close to the surface. The observed divergence of $\left.C_{n}^{2}\right|_{x}$ and $\left.C_{n}^{2}\right|_{y}$ near the surface is apparently associated with the flow anisotropy resulting from surface wind shear. 

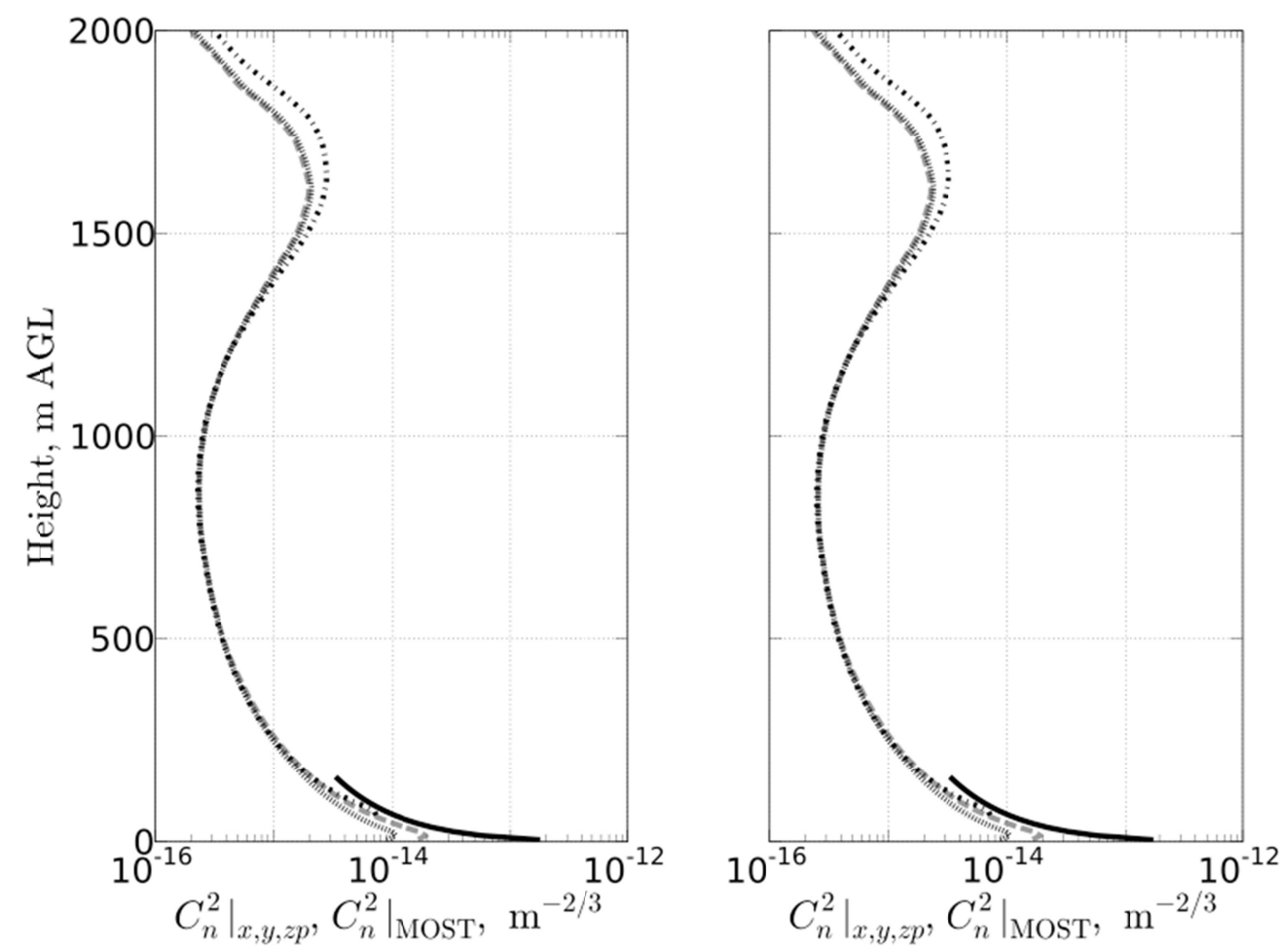

Figure 4: Comparison of the vertical profile of $\left.C_{n}^{2}\right|_{x}$ (dashed line), $\left.C_{n}^{2}\right|_{y}$ (dotted line), $\left.C_{n}^{2}\right|_{z}$ (dashed-dotted line) and MOST calculations (solid line).

\section{CONCLUSIONS}

Feasibility of direct evaluation of the refractive index structure function $C_{n}^{2}$ and structure function parameters $C_{T}^{2}, C_{q}^{2}$, and $C_{T q}$ from the output of large eddy simulations (LES) for the atmospheric convective boundary layer (CBL) has been investigated. One considered approach involved the direct evaluation of $C_{n}^{2}$ from the simulated refractive index field. The second approach was based on a parameterization of $C_{n}^{2}$ in terms of the structure-function parameters for temperature and humidity which, in turn, were retrieved from the LES fields of potential temperature and specific humidity.

Both methods yield refractive index structure functions that follow the inertial-subrange 2/3-law for a fair range of the separation distances in the well-mixed portion of the CBL. The lower limit of the separation distance depends on the LES spatial resolution and appears to be approximately four times the LES grid spacing. The upper limit of the separation distance for the spatial averaging in $z$-direction depends on the elevation with the CBL: deviation from the 2/3-law is noted when spatial averaging takes place for two points that belong to portions of the CBL flow with distinctively different turbulence properties.

The $C_{n}^{2}$ results yielded by the two LES-based approaches are very similar. In the lower CBL portion they also fairly agree with $C_{n}^{2}$ values predicted by the Monin-Obukhov similarity theory (MOST). In this respect, the $C_{n}^{2}$ retrieval from the LES data is promising for evaluating the vertical profile of $C_{n}^{2}$ throughout the entire CBL.

Under the considered CBL conditions and for the selected optical wavelength of $0.55 \mu \mathrm{m}$ the value of $C_{n}^{2}$ was found to be dominated by the $C_{T}^{2}$ contribution in the first few hundred meters above the surface, whereas the $C_{T q}$ contribution became significant aloft. 


\section{ACKNOWLEDGEMENTS}

The first and third co-authors gratefully acknowledge support provided for a part of this study by the National Science Foundation, USA, through the grant ATM-1016153.

\section{REFERENCES}

[1] Burk, S.D., "Refractive index structure parameters: time-dependent calculations using a numerical boundarylayer model," J. Appl. Meteor. 19, 562-576 (1980).

[2] Cheinet, S. and Siebesma, A.P., "Variability of local structure parameters in the convective boundary layer: a large-eddy simulation analysis," J. Appl. Meteor. Climatol. 50, 472-481 (2009).

[3] De Jong, A.N., "Enhanced IR point target detection by atmospheric effects," Proc. SPIE 4820, 885 (2003)

[4] Andreas, E.L., "Estimating Cn2 over snow and sea ice from meteorological data," J. Opt. Soc. Am. A. 5, 481495 (1988).

[5] Tatarskii, V.I., [Wave propagation in a turbulent medium], McGraw-Hill, New York (1961).

[6] Monin, A.S., and Yaglom, A.M., [Statistical fluid mechanics: mechanics of turbulence, vol. 2], MIT Press, Cambridge MA (1975).

[7] Peltier, L.J., and Wyngaard, J.C., "Structure-function parameters in the convective boundary layer from largeeddy simulation," J. Atmos. Sci. 52, 3641-3360 (1995).

[8] Wyngaard, J.C., Pennell, W.T., Lenschow, D.H., and LeMone, M.A., "The temperature-humidity covariance budget in the convective boundary layer,", J. Atmos. Sci. 35, 47-58 (1978).

[9] Beland, R.R., "Propagation through Atmospheric Optical Turbulence," [The Infrared \& Electro-Optical Systems Handbook: Atmospheric Propagation of Radiation vol. 2], Infrared Information Analysis Center, Ann Arbor, Michigan 48113-4001 and SPIE Optical Engineering Press, Bellingham, WA, USA, 157-232 (1993).

[10] Kaimal, J.C., Wyngaard, J.C., Haugen, D.A., Cote, O.R., and Izumi, Y., "Turbulence structure in the convective boundary layer," J. Atmos. Sci. 33, 2152-2169 (1976).

[11] Murphy, E.A., Dewan, E.M., and Sheldon, S.M., "Daytime comparisons of Cn2 models to measurements in a desert location," Proc. SPIE 551, 156-162 (1985).

[12] Hufnagel, R.E., "Propagation through atmospheric turbulence," [The infrared handbook], Environmental research institute of Michigan, chapter 6 (1974).

[13] Valley, G.C., "Isoplanatic degradation of tilt correction and short-term imaging systems," Appl. Opt. 19, 574577 (1980).

[14] Panofsky, H.A., and Dutton, J.A., [Atmospheric turbulence: models and methods for engineering applications], John Wiley \& Sons, New York, USA (1984)

[15] Wyngaard, J.C., Izumi, Y., and Collins Jr, S.A., "Behavior of the refractive index structure parameter near the ground," J. Opt. Soc. Am. 61, 1646-1650 (1971).

[16]Fairall, C.W., Schacher, G.E., and Davidson, K.L., "Measurements of the humidity structure function parameters Cq2 and CTq, over the ocean," Boundary-Layer Meteorol. 19, 81-92 (1980).

[17] Kohsiek, W., "Measuring CT2, CQ2 and CTQ in the unstable surface layer, and relations to the vertical fluxes of heat and moisture," Boundary-Layer Meteorol. 24, 89-107 (1982).

[18] Wang, T.I., Ochs, G.R., and Clifford, S.F., “A saturation-resistant optical scintillometer to measure $\mathrm{C}_{\mathrm{n}}^{2}$," J. Opt. Soc. Am. 69, 334-338 (1978).

[19] The Weather Research \& Forecasting model, <http://www.wrf-model.org/wrfadmin/publications.php >

[20] Gibbs, J.A., Fedorovich, E., and Van Eijk, A.M.J., "Evaluating Weather Research and Forecasting (WRF) model predictions of turbulent flow parameters in a dry convective boundary layer," J. Appl. Meteorol. Clim. 50, 2429-2444 (2011).

[21] Smagorinsky, J., "General circulation experiments with primitive equations," Mon. Wea. Rev. 91, 99-164 (1963)

[22] Deardorff, J.W., “A numerical study of three-dimensional turbulent channel flow at large Reynolds numbers," J. Fluid Mech. 41, 453-480 (1970).

[23] Wilson, C., and Fedorovich, E., "Direct evaluation of refractive-index structure functions from large-eddy simulation output for atmospheric convective boundary layers," Acta Geophys. 60, 1474-1492 (2012). 
[24]Fedorovich, E., Conzemius, R., and Mironov, D., "Convective entrainment into a shear-free, linearly stratified atmosphere: Bulk models reevaluated through large eddy simulations," J. Atmos. Sci. 61, 281-295 (2004)

[25]Fedorovich, E., Conzemius, R., Esau, I., Katopodes-Chow, F., Lewellen, D., Moeng, C-H., Pino, P., Sullivan, P., and Villa-Guerau de Arellano, J., "Entrainment into shared convective boundary layers as predicted by different large eddy simulations," [16 ${ }^{\text {th }}$ symposium on boundary layers and turbulence], American Meteorological Scoiety, Portland, ME USA, P.4.7 (2004).

[26] Cook, D.R., and Pekour, M.S., [Eddy correlation flux measurement systems handbook], Tech. Rep. DOE/SCARM/TR-05, 15 pp (2008).

[27] Benjamin, S.G., Brundage, K.J., and Morone, L.L., [Implementation of the Rapid Update Cycle. Part 1: Analysis/Model description], NOAA/NWS Technical Procedures Bull., 16 pp (1994)

[28] Frederickson, P.A., Davidson, K.L., Zeisse, C.R., and Bendall, C.S., "Estimating the refractive index structure parameter (Cn2) over the ocean using bulk methods," J. Appl. Meteor. 39, 1770-1783 (2000). 